\title{
THE THEORY OF WAGE DIFFERENTIALS: PRODUCTION RESPONSE AND FACTOR PRICE EQUALISATION
}

\author{
Jagdish N.BIIAGWATI \\ Department of Economics, Massachusetts \\ Institute of Technology, Cambridge, \\ Mass. 02139
}

\author{
T.N. SRINIVASAN \\ Indian Statistical Institute, \\ Planning Unit
}

Previous analyses of the case where there is a distortionary wage differential between different activities, by writers such as Fishlow and David (1961), Hagen (1958), Bhagwati and Ramaswami (1963), and Johnson (1966) have led to the discovery of the following pathologies:

(i) the feasible production possibility curve will shrink inside the "best" production possibility curve (Hagen, 1958);

(ii) the commodity-price ratio will not be tangential (at points of incomplete specialisation in production) to the feasible production possibility curve (Hagen, 1958); and

(iii) the production possibility curve may become convex to the origin, instead of concave (Fishlow and David, 1961; Bhagwati and Ramaswami, 1963; and Johnson, 1966).

However, this is not the end of the story. It can be further shown that (i) the shift in the production of a commodity, as its relative price changes, may be either positive or negative; and that the shift is not necessarily predictable from the convexity or concavity of the feasible production possibility curve; and (ii) given the commodity-price ratio, we cannot necessarily have unique capital-labour ratios in the two activities or unique factor price ratios: an important and interesting implication of which result is that the factor price-equalisation theorem breaks down, despite all the Samuelson conditions being met, even if there is an identical wage differential in the same sector in both countries.

We would like to note that research in the field of wage differentials is independently being conducted by Steve Magee, P.J.Lloyd, Ronald Jones, and by Murray Kemp and Horst Herberg. Thanks are due to Harry Johnson and Ronald Jones for comments on an earlier draft of the paper. The research of the former author has been supported by a grant from the National Science Foundation. 


\section{The model}

We consider the standard two-factor, two-commodity model of trade theory. The production function for each commodity will be assumed to be homogeneous of degree one, and strictly concave with nonnegative marginal products for both factors. Each factor is limited in availability and supplied inelastically to the extent of availability. The price paid for the use of one of the factors in the production of one of the commodities is, however, assumed to be a given constant times the price paid for the use of the same factor in the production of the other commodity. Given the international commodity-price ratio, production is assumed to take place under purely competitive conditions.

Let $R_{i}$ denote the factor intensity (ratio of the amount of the first factor to that of the second) in the production of commodity $i$. Let $R$ be the aggregate factor endowment ratio. Without loss of generality, we shall assume that the total availability of the second factor is unity. Let $L$ be the amount of the second factor employed in the production of the first commodity. Let $f^{i}\left(R_{i}\right)$ be the average physical product of the second factor in the production of commodity $i$ when the factorintensity is $R_{i}$. Let $Q_{i}$ be the output of commodity $i$. Let $\gamma$ be the ratio of the reward of the second factor in the production of the second commodity to that in the production of the first commodity. Let $f_{1}^{i}\left(R_{i}\right)$ be the derivative of $f^{i}\left(R_{i}\right)$ with respect to $R_{i}$. Clearly $f_{1}^{i}\left(R_{i}\right)$ is the marginal physical product of the first factor in the production of commodity $i$. Let $p$ be the international price of the second commodity in terms of the first.

Given our assumptions we can now describe our model algebraically as follows:

$$
\begin{aligned}
& Q_{1}=L f^{1}\left(R_{1}\right), \\
& Q_{2}=(1-L) f^{2}\left(R_{2}\right), \\
& L R_{1}+(1-L) R_{2}=R, \\
& f_{1}^{1}\left(R_{1}\right)=p f_{1}^{2}\left(R_{2}\right), \\
& \left\{f^{1}-R_{1} f_{1}^{1}\left(R_{1}\right)\right\} \gamma=p\left\{f^{2}-R_{2} f_{1}^{2}\left(R_{2}\right)\right\} .
\end{aligned}
$$


Eqs. (1), (2), and (3) represent the production functions and factor allocations. Eq. (4) states that the reward of the first factor (i.e. its marginal value product) is the same in the production of either commodity. Eq. (5) states that the reward of the second factor in the production of the second commodity is $\gamma$ times its reward in the production of the first.

\section{The comparative statics of equilibrium outputs}

In order now to investigate the response of output of either of the two commodities as the commodity-price ratio changes, it is convenient to work in terms of the variable $w$ representing the ratio of the reward of the second factor to that of the first in the production of the first commodity. Then we can write:

$$
\begin{aligned}
& \frac{f^{1}-R_{1} f_{1}^{1}}{f_{1}^{1}}=w, \\
& \frac{f^{2}-R_{2} f_{1}^{2}}{f_{1}^{2}}=\gamma w .
\end{aligned}
$$

Given our concavity assumptions we can solve (6) and (7) uniquely ${ }^{1}$ to obtain $R_{1}$ and $R_{2}$ as functions $R_{1}(w)$ and $R_{2}(w)$ of $w$. It is easily seen that $R_{i}(w)$ is an increasing function of $w$. Given $R$, let $w_{i}(R)$ be the unique solution of $R_{i}(w)=R$. Then the relevant range of values for $w$ is the interval [ $\underline{\mathbf{w}}, \bar{w}]$ where $\underline{\mathbf{w}}(\bar{w})$ is the smaller (larger) of $w_{1}(R)$ and $w_{2}(R)$ (see fig. 1). The value of $L$ corresponding to any given $w$ in this interval is obtained from eq. (3). The equilibrium value (or values) of $w$ corresponding to a given $p$ is (are) obtained from eq. (4).

Let us examine this equation more closely. Let us first rewrite it as:

$$
\frac{f_{1}^{1}\left(R_{1}\right)}{f_{1}^{2}\left(R_{2}\right)}=p
$$

${ }^{1}$ If we wish to ensure that a solution exists for all non-negative values of $w$, we have to assume the Inada conditions:

$$
\lim _{R_{i} \rightarrow 0(\infty)} \frac{f^{i}-R_{i} f_{1}^{i}\left(R_{i}\right)}{f_{1}^{i}\left(R_{i}\right)}=0(\infty) .
$$




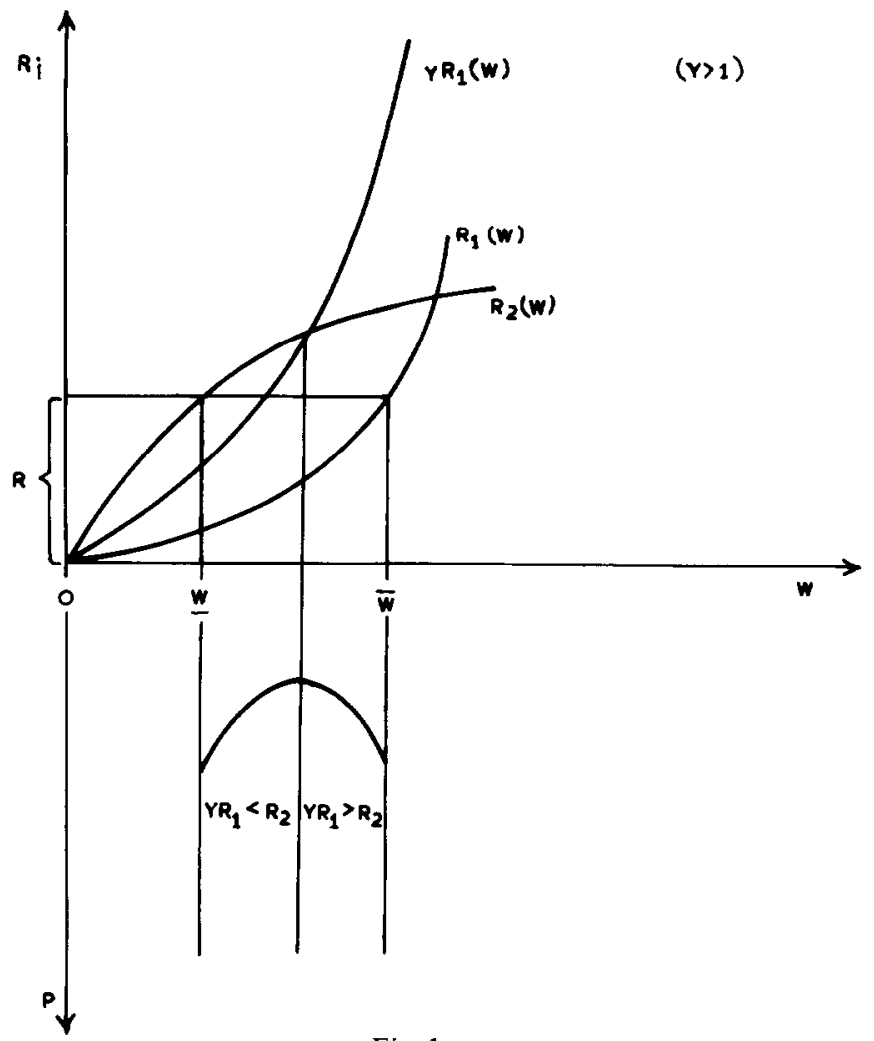

Fig. 1.

The left-hand side of eq. (8) is a function of $w$ alone. Denoting this function by $p(w)$ we get:

$$
\frac{p^{\prime}(w)}{p(w)}=\frac{1}{p(w)} \frac{\mathrm{d} p(w)}{\mathrm{d} w}=\frac{f_{11}^{1}\left(R_{1}\right)}{f_{1}^{1}\left(R_{1}\right)} \frac{\mathrm{d} R_{1}(w)}{\mathrm{d} w}-\frac{f_{11}^{2}\left(R_{2}\right)}{f_{1}^{2}\left(R_{2}\right)} \cdot \frac{\mathrm{d} R_{2}(w)}{\mathrm{d} w}
$$

where $f_{11}^{i}\left(R_{i}\right)$ is $\left[\mathrm{d}^{2} f^{i}\left(R_{i}\right)\right] / \mathrm{d} R_{i}^{2}$

From (6) and (7) we get:

$$
\begin{aligned}
& -\frac{f_{11}^{1}\left(R_{1}\right) f^{1}\left(R_{1}\right)}{\left\{f_{1}^{1}\left(R_{1}\right)\right\}^{2}} \cdot \frac{\mathrm{d} R_{1}(w)}{\mathrm{d} w}=1, \\
& -\frac{f_{11}^{2}\left(R_{2}\right) f^{2}\left(R_{2}\right)}{\left\{f_{1}^{2}\left(R_{2}\right)\right\}^{2}} \cdot \frac{\mathrm{d} R_{2}(w)}{\mathrm{d} w}=\gamma .
\end{aligned}
$$


Hence

$$
\begin{aligned}
\frac{p^{\prime}(w)}{p(w)} & =-\frac{f_{1}^{1}\left(R_{1}\right)}{f^{1}\left(R_{1}\right)}+\frac{f_{1}^{2}\left(R_{2}\right)}{f^{2}\left(R_{2}\right)} \\
& =-\frac{1}{w+R_{1}}+\frac{\gamma}{\gamma w+R_{2}} \quad[\operatorname{using}(6) \text { and }(7)] \\
& =\frac{\left(\gamma R_{1}-R_{2}\right)}{\left(w+R_{1}\right)\left(\gamma w+R_{2}\right)} .
\end{aligned}
$$

Eq. (11) points at once to an interesting set of possibilities.

First, if in the relevant interval $[\underline{w}, \bar{w}]$ of values of $w,\left[\gamma R_{1}(w)-R_{2}(w)\right]$ changes sign, then $p^{\prime}(w)$ also changes sign, since $w+R_{1}(w)$ and $\gamma w+R_{2}(w)$ are both non-negative. In other words, $p(w)$ is not a monotonic function of $w$. Thus eq. (8) can have more than one value of $w$ in $[\underline{w}, \bar{w}]$ as a solution. This means that the same commodity-price ratio $p$ can be consistent with more than one equilibrium combination of the outputs of the two commodities.

Second, consider two countries with identical production functions and the same type and degree of distortion (i.e. the second factor in the production of the second commodity receives $\gamma$ times its reward in the production of the first commodity in both countries). Suppose they face the same commodity-price ratio $p$. If $\gamma R_{1}(w)-R_{2}(w)$ changes sign in both countries within the respective interval of values of $w$, then one country's equilibrium value $w$ could be different from that of the other. In other words, factor price equalisation will fail to take place. It is important to note that this failure could take place, even though there is no factor-intensity reversal in the usual sense: even though $\left[R_{1}(w)-R_{2}(w)\right]$ has the same sign for all relevant values of $w$, for both countries, $\left[\gamma R_{1}(w)-R_{2}(w)\right]$ can still change its sign. ${ }^{2}$

The precise conditions under which such multiple equilibria will arise can be readily derived and related to the conditions defining the nature of output response to price change. To do this, we proceed now to

\footnotetext{
2 It should of course be kept in mind that $R_{2}(w)$ is the factor intensity in the production of the second commodity when the factor price ratio faced by producers of this commodity is $\gamma w$.
} 
derive first the slope of the production possibility curve, given $\gamma$. Using (1), (2), and (3), we get:

$$
\begin{aligned}
& \frac{\mathrm{d} Q_{1}}{\mathrm{~d} w}=\frac{\mathrm{d} L}{\mathrm{~d} w} f^{1}+L f_{1}^{1} \frac{\mathrm{d} R_{1}}{\mathrm{~d} w}, \\
& \frac{\mathrm{d} Q_{2}}{\mathrm{~d} w}=-\frac{\mathrm{d} L}{\mathrm{~d} w} f^{2}+(1-L) f_{1}^{2} \frac{\mathrm{d} R_{2}}{\mathrm{~d} w}, \\
& \frac{\mathrm{d} L}{\mathrm{~d} w}=\frac{\left(R_{2}-R\right)\left(\mathrm{d} R_{1} / \mathrm{d} w\right)+\left(R-R_{1}\right)\left(\mathrm{d} R_{1} / \mathrm{d} w\right)}{\left(R_{2}-R_{1}\right)^{2}} .
\end{aligned}
$$

Hence

$$
\begin{aligned}
\frac{\mathrm{d} Q_{1}}{\mathrm{~d} w} & =\frac{f^{2}\left\{\left(R_{2}-R\right) \frac{\mathrm{d} R_{1}}{\mathrm{~d} w}+\left(R-R_{1}\right) \frac{\mathrm{d} R_{2}}{\mathrm{~d} w}\right\}+f_{1}^{1}\left(R_{2}-R\right)\left(R_{2}-R_{1}\right) \frac{\mathrm{d} R_{1}}{\mathrm{~d} w}}{\left(R_{2}-R_{1}\right)^{2}} \\
& =\frac{\left\{\left(f^{1}-R_{1} f_{1}^{1}\right)+R_{2} f_{1}^{1}\right\}\left(R_{2}-R\right) \frac{\mathrm{d} R_{1}}{\mathrm{~d} w}+f^{1}\left(R-R_{1}\right) \frac{\mathrm{d} R_{2}}{\mathrm{~d} w}}{\left(R_{2}-R_{1}\right)^{2}} \\
& =\frac{f_{1}^{1}\left[\left\{\left(\frac{f^{1}-R_{1} f_{1}^{1}}{f_{1}^{1}}\right)+R_{2}\right\}\left(R_{2}-R\right) \frac{\mathrm{d} R_{1}}{\mathrm{~d} w}+\frac{f^{1}}{f_{1}^{1}}\left(R-R_{1}\right) \frac{\mathrm{d} R_{2}}{\mathrm{~d} w}\right]}{\left(R_{2}-R_{1}\right)^{2}} \\
& =\frac{f_{1}^{1}\left\{\left(w+R_{2}\right)\left(R_{2}-R\right) \frac{\mathrm{d} R_{1}}{\mathrm{~d} w}+\left(w+R_{1}\right)\left(R-R_{1}\right) \frac{\mathrm{d} R_{2}}{\mathrm{~d} w}\right\}}{\left(R_{2}-R_{1}\right)^{2}} \text { [using (6)] }
\end{aligned}
$$

and

$$
\frac{\mathrm{d} Q_{2}}{\mathrm{~d} w}=\frac{f_{1}^{2}\left\{\left(\gamma w+R_{2}\right)\left(R_{2}-R\right) \frac{\mathrm{d} R_{1}}{\mathrm{~d} w}+\left(\gamma w+R_{1}\right)\left(R-R_{1}\right) \frac{\mathrm{d} R_{2}}{\mathrm{~d} w}\right\}}{\left(R_{2}-R_{1}\right)^{2}}
$$


Now:

$$
\frac{\mathrm{d} R_{1}}{\mathrm{~d} w}=\frac{-\left(f_{1}^{1}\right)^{2}}{f_{11}^{1} f^{1}} \quad \text { and } \quad \frac{\mathrm{d} R_{2}}{\mathrm{~d} w}=\frac{-\left(f_{1}^{2}\right)^{2} \gamma}{f_{11}^{2} f^{2}}
$$

Next, let $\sigma\left(R_{i}\right)$ denote the elasticity of substitution of the factors in the production of the $i$ th commodity. It is well known that

$$
\sigma\left(R_{i}\right)=-\frac{f_{1}^{i}\left(f^{i}-R_{i} f_{1}^{i}\right)}{R_{1} f^{i} f_{11}^{i}}
$$

Using (6) and (7) therefore we can write:

$$
\begin{aligned}
& \frac{\mathrm{d} Q_{1}}{\mathrm{~d} w}=\frac{f_{1}^{1}\left\{\left(w+R_{2}\right)\left(R_{2}-R\right) \sigma_{1} R_{1}+\left(w+R_{1}\right)\left(R-R_{1}\right) \sigma_{2} R_{2}\right\}}{w\left(R_{2}-R_{1}\right)^{2}} \\
& \frac{\mathrm{d} Q_{2}}{\mathrm{~d} w}=\frac{-f_{1}^{2}\left\{\left(\gamma w+R_{2}\right)\left(R_{2}-R\right) \sigma_{1} R_{1}+\left(\gamma w+R_{1}\right)\left(R-R_{1}\right) \sigma_{2} R_{2}\right\}}{w\left(R_{2}-R_{1}\right)^{2}}
\end{aligned}
$$

Hence

$$
\begin{aligned}
\frac{\mathrm{d} Q_{1}}{\mathrm{~d} Q_{2}} & =-\frac{f_{1}^{1}}{f_{1}^{2}}\left\{\frac{\left(w+R_{2}\right)\left(R_{2}-R\right) \sigma_{1} R_{1}+\left(w+R_{1}\right)\left(R-R_{1}\right) \sigma_{2} R_{2}}{\left(\gamma w+R_{2}\right)\left(R_{2}-R\right) \sigma_{1} R_{1}+\left(\gamma w+R_{1}\right)\left(R-R_{1}\right) \sigma_{2} R_{2}}\right\} \\
& =-p\left\{\frac{\left(w+R_{2}\right)\left(R_{2}-R\right) \sigma_{1} R_{1}+\left(w+R_{1}\right)\left(R-R_{1}\right) \sigma_{2} R_{2}}{\left(\gamma w+R_{2}\right)\left(R_{2}-R\right) \sigma_{1} R_{1}+\left(\gamma w+R_{1}\right)\left(R-R_{1}\right) \sigma_{2} R_{2}}\right\} .
\end{aligned}
$$

It is seen from (14) that if there is no distortion, i.e. if $\gamma=1$, then $\mathrm{d} Q_{1} / \mathrm{d} Q_{2}=-p$, showing that the domestic rate of transformation $\left(-\mathrm{d} Q_{1} / \mathrm{d} Q_{2}\right)$ equals the commodity-price ratio $p$. However, if $\gamma \neq 1$, we see from (14) that:

$$
\begin{aligned}
& p-\left(-\frac{\mathrm{d} Q_{1}}{\mathrm{~d} Q_{2}}\right) \\
& =p(\gamma-1) w\left\{\frac{\left(R_{2}-R\right) \sigma_{1} R_{1}+\left(R-R_{1}\right) \sigma_{2} R_{2}}{\left(\gamma w+R_{2}\right)\left(R_{2}-R\right) \sigma_{1} R_{1}+\left(\gamma w+R_{1}\right)\left(R-R_{1}\right) \sigma_{2} R_{2}}\right\} .
\end{aligned}
$$


The expression in the square parentheses is always positive; hence $p \gtreqless-\mathrm{d} Q_{1} / \mathrm{d} Q_{2}$ according as $\gamma \gtreqless 1$. This means that the commodityprice ratio, $p$, will not equal and will indeed exceed (fall short of) the domestic rate of transformation $\left(-\mathrm{d} Q_{1} / \mathrm{d} Q_{2}\right)$ according as the degree of distortion in the factor price ratio faced by the producers of the second commodity as compared to those of the first, i.e. $\gamma$, exceeds (falls short of) unity.

Note further that, in general, the degree of divergence between the commodity-price ratio and the marginal rate of transformation can be expected to vary with the equilibrium point on the production possibility curve at which this divergence is being measured (although this is not inevitable). ${ }^{3}$ It is also clear that this variation in the degree of divergence can obtain under CES production functions (where $\sigma_{1}$ and $\sigma_{2}$ are constant) and even under Cobb-Douglas production functions (where $\sigma_{1}=\sigma_{2}=1$ ). Furthermore, the possibility of multiple equilibria which we have already noted, also implies that, corresponding to the same commodity-price ratio, there could be different divergences between the price ratio and the marginal rate of transformation at alternative equilibirum points on the production possibility curve.

We are now in a position to examine the response of the equilibirum output $Q_{2}$ of the second commodity to change in the international price ratio $p$. From (13), it is clear that $\mathrm{d} Q_{2} / \mathrm{d} w \gtreqless 0$ according as $R_{2}(w) \gtreqless R_{1}(w)$. By definition (except in the trivial case where $\underline{\mathbf{w}}=\bar{w}$, either $R_{2}(w)>R_{1}(w)$ or $R_{2}(w)<R_{1}(w)$ for all $w$ in $[\underline{\mathbf{w}}, \bar{w}]$. Since the interval $[\underline{\mathbf{w}}, \bar{w}]$ is determined uniquely once the aggregate factor endowment is known, the sign of $\mathrm{d} Q_{2} / \mathrm{d} w$ is also uniquely determined. To get the response of $Q_{2}$ with respect to $p$ we have to evaluate $\mathrm{d} Q_{2} / \mathrm{d} p=\left(\mathrm{d} Q_{2} / \mathrm{d} w\right) \cdot(\mathrm{d} w / \mathrm{d} p)$. Remember also that $\mathrm{d} w / \mathrm{d} p$ will have the same sign as $p^{\prime}(w)$ in eq. (11); and that $p^{\prime}(w) \gtreqless 0$ according as $\left[\gamma R_{1}-R_{2}\right] \gtreqless 0$. Using these arguments, we can now proceed to analyse the following six cases (excluding the degenerate case of $\underline{\mathbf{w}}=\bar{w}$ ):

Case I: $R_{1}(w)>R_{2}(w)$ and $\gamma R_{1}(w)>R_{2}(w)$ for all $w$ in $[\underline{\mathbf{w}}, \bar{w}]$. Given $R_{1}(w)>R_{2}(w)$, this case will arise when either $\gamma \geq 1$ or when $\gamma$ is less than unity but not sufficiently less than unity to make $\gamma R_{1}(w)$

\footnotetext{
${ }^{3}$ This can be seen readily by dividing (14a) on both sides by ' $p$ ', which yields the formula for $-p /\left(\mathrm{d} Q_{1} / \mathrm{d} Q_{2}\right)$, the relative degree of divergence. Note also that, except for the multipleequilibrium possibility discussed in the text, any movement along the production possibility curve in equilibrium will require a change in the commodity-price ratio.
} 
less than $R_{2}(w)$ for some $w$ in [w, $\bar{w}$ ]. In this case, $\mathrm{d} Q_{2} / \mathrm{d} w>0$ and assuming incomplete specialisation $\mathrm{d} w / \mathrm{d} p>0$. Hence $\mathrm{d} Q_{2} / \mathrm{d} p>0$. Thus, if we compare the equilibrium output $Q_{2}$ corresponding to two different international price ratios, the one associated with the higher price of the second commodity in terms of the first will be larger. Thus the (comparative static) response of equilibrium output to a price change is 'normal'.

Case II: $R_{1}(w)<R_{2}(w)$ and $\gamma R_{1}(w)<R_{2}(w)$ for all $w$ in $[\underline{\mathbf{w}}, \bar{w}]$. Given $R_{1}(w)<R_{2}(w)$, this case will arise when either $\gamma \leq 1$ or when $\gamma$ is greater than unity but not sufficiently greater to make $\gamma R_{1}(w)$ exceed $R_{2}(w)$ for some $w$ in $[\underline{\mathbf{w}}, \bar{w}]$. In this case $\mathrm{d} Q_{2} / \mathrm{d} w<0$ and again assuming incomplete specialisation $\mathrm{d} w / \mathrm{d} p<0$. Hence $\mathrm{d} Q_{2} / \mathrm{d} p>0$. Thus the output response is again 'normal'.

Case III: $R_{1}(w)>R_{2}(w)$ but $\gamma R_{1}(w)<R_{2}(w)$ for all $w$ in [w, $\bar{w}$ ]. Given $R_{1}(w)>R_{2}(w)$ this can happen only when $\gamma$ is sufficiently less than unity. In this case $\mathrm{d} Q_{2} / \mathrm{d} w>0$ and, assuming incomplete specialisation, $\mathrm{d} w / \mathrm{d} p<0$. Hence $\mathrm{d} Q_{2} / \mathrm{d} p<0$. Thus if we compare the equilibrium outputs corresponding to two different international prices for the second commodity in terms of the first, then the output corresponding to a higher price will be smaller. This is a case of 'perverse' comparative-static response.

Case IV: $R_{1}(w)<R_{2}(w)$ but $\gamma R_{1}(w)>R_{2}(w)$ for all $w$ in [w, $\bar{w}$ ]. Given $R_{1}(w)<R_{2}(w)$, this can arise only when $\gamma$ is sufficiently greater than unity. In this case also the output response in a comparative-static sense is 'perverse'.

Case V: $R_{1}(w)>R_{2}(w)$ but $\gamma R_{1}(w)-R_{2}(w)$ changes sign at one or more $w$ in $[\underline{\mathbf{w}}, \bar{w}]$. We saw earlier than when $\gamma R_{1}(w)-R_{2}(w)$ changes sign in $[\underline{\mathbf{w}}, \bar{w}]$, the same international price ratio $p$ may correspond to more than one equilibrium value for $w$ and hence for the outputs $Q_{1}$ and $Q_{2}$. Thus the derivative $\mathrm{d} w / \mathrm{d} p$ will be different depending on the particular equilibrium value of $w$ at which it is evaluated. Hence the sign of $\mathrm{d} Q_{2} / \mathrm{d} p$ will depend on the particular equilibrium point at which it is evaluated. It is easy to see that if we order the equilibrium points in increasing order of the value of $Q_{2}$ then $\mathrm{d} Q_{2} / \mathrm{d} p$ will alternate in sign as we move from one equilibrium point to the next. Thus if at one equilibrium point the comparative static response is 'normal', then at the next it will be 'perverse'. 
Case VI: $R_{1}(w)<R_{2}(w)$ but $\gamma R_{1}(w)-R_{2}(w)$ changes sign at one or more $w$ in [ $\underline{\mathbf{w}}, \bar{w}]$. Here again the possibility of multiple equilibria arises and the conclusions in Case V apply to this case also.

We may finally note that these results can be readily translated into, and derived via, the familiar Lerner technique as revived by Findlay and Grubert (1959). Thus, for example, the central result of our analysis, which demonstrates the possibility of multiple $w$-values corresponding to a single $p$-value, can be illustrated in terms of the Lerner technique as follows.

In fig. 2, the commodity-price ratio involves an exchange of 1 for $\overline{2}$ units of the two commodities. Then, it is easy to see that, consistent with commodity 1 remaining intensive in the use of factor 2 in two alternative equilibria (i.e. $\mathrm{OM}^{2}$ is steeper than $\mathrm{OM}^{1}$, and so is $\mathrm{ON}^{2}$ steeper than $\mathrm{ON}^{1}$ ), two alternative values of the factor price ratio are possible when the commodity-price ratio is fixed at an exchange of 1 for $\overline{2}$ units of the two commodities. These two alternative factor price ratios are: $[\Lambda \mathrm{C}, \mathrm{AB}]$ and $[\mathrm{DF}, \mathrm{DE}]$ (such that the wage of factor 2 in commodity 2 is higher in each case by the same multiplicative factor than in commodity 1 ).

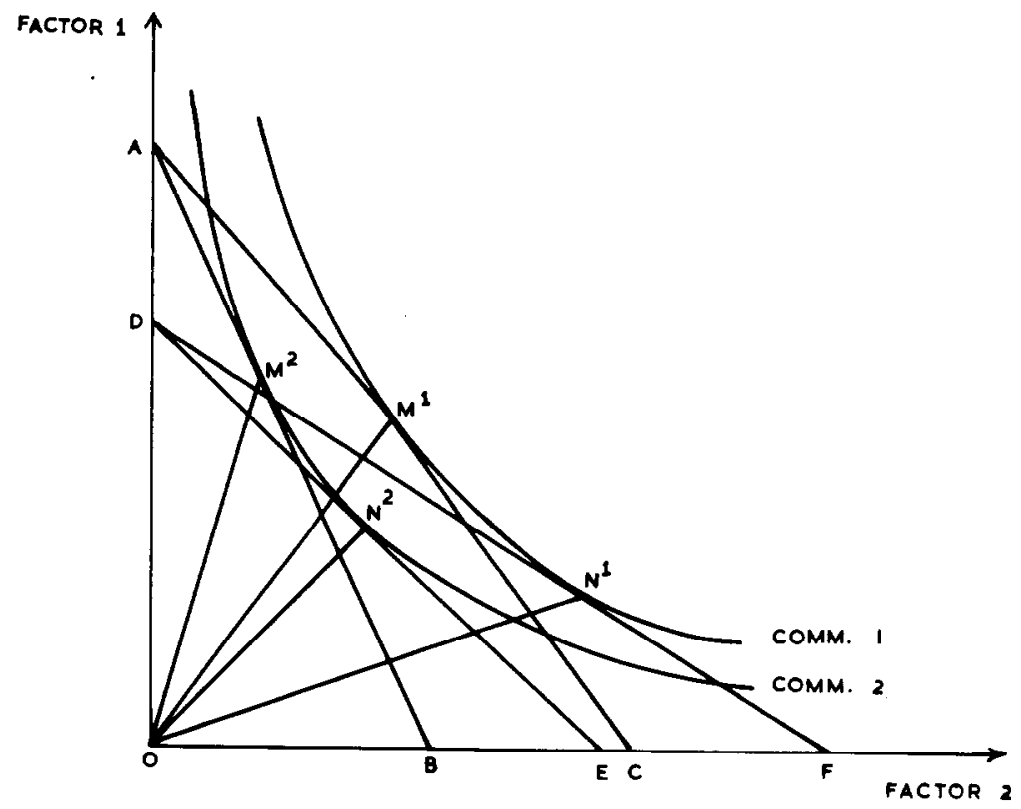

Fig. 2. 


\section{Relationship of output response to shape of the production possibili- ty curve}

The possibility of 'perverse' production response to change in the commodity-price ratio, in the presence of the wage differential, raises in turn the question as to whether the 'perverse' response will arise if and only if the production possibility curve is convex to the origin.

Such an inference is implicit in the earlier literature (Bhagwati and Ramaswami, 1963; Hagen, 1958), in the way the diagrams are drawn, for example, to show that the output of a commodity increases with its relative price when the production possibility curve is concave to the origin. However, such an inference is logically valid only when there is no wage differential. In the absence of such a differential the commodity-price ratio will be tangential to the production possibility curve and hence the output response to price change depends entirely on the curvature of this curve. But, once the differential is present, the commodity-price ratio no longer equals the domestic rate of transformation and hence there is no a priori rcason to expect any necessary connection between output response and the curvature of the production possibility curve. Our numerical example in the Appendix does in fact show that there is no such connection. However, it is nevertheless of interest to derive analytically the curvature of the production possibility curve. To this we now turn. We showed (eq. (14)) that:

$$
\frac{\mathrm{d} Q_{1}}{\mathrm{~d} Q_{2}}=-\frac{f_{1}^{1}}{f_{1}^{2}}\left[\frac{\left(w+R_{2}\right)\left(R_{2}-R\right) \sigma_{1} R_{1}+\left(w+R_{1}\right)\left(R-R_{1}\right) \sigma_{2} R_{2}}{\left(\gamma w+R_{2}\right)\left(R_{2}-R\right) \sigma_{1} R_{1}+\left(\gamma w+R_{1}\right)\left(R-R_{1}\right) \sigma_{2} R_{2}}\right]
$$

We can then derive $\mathrm{d}^{2} Q_{1} / \mathrm{d} Q_{2}^{2}$ by using the relation $\mathrm{d}^{2} Q_{1} / \mathrm{d} Q_{2}^{2}=$ $\mathrm{d}\left[\mathrm{d} Q_{1} / \mathrm{d} Q_{2}\right] / \mathrm{d} w . \mathrm{d} w / \mathrm{d} Q_{2}$ and $\mathrm{d} w / \mathrm{d} Q_{2}=1 /\left(\mathrm{d} Q_{2} / \mathrm{d} w\right)$. We have already derived the expression for $\mathrm{d} Q_{2} / \mathrm{d} w$ in eq. (13). Let us denote the numerator of the detailed expression for $\mathrm{d} Q_{1} / \mathrm{d} Q_{2}$, with the negative sign, by $N(w)$ and the denominator by $D(w)$. Then

$$
\frac{\mathrm{d}}{\mathrm{d} w} \frac{\mathrm{d} Q_{1}}{\mathrm{~d} Q_{2}}=\frac{D(w) \frac{\mathrm{d} N}{\mathrm{~d} w}-N(w) \frac{\mathrm{d} D}{\mathrm{~d} w}}{D^{2}} .
$$


Now, using the relations $\mathrm{d} R_{i} / \mathrm{d} w=\sigma_{i} R_{i} / w$, we can show that:

$$
\begin{aligned}
\frac{\mathrm{d} N}{\mathrm{~d} w}= & -\left[\left(w+R_{2}\right)\left(R_{2}-R\right) \sigma_{1} R_{1}+\left(w+R_{1}\right)\left(R-R_{1}\right) \sigma_{2} R_{2}\right] \\
& \times \frac{f_{1}^{1}}{w}\left[\frac{\sigma_{1} R_{1} f_{11}^{1}}{f_{1}^{1}}+\left(\sigma_{1}+\sigma_{2}\right)\right]-f_{1}^{1}\left[\left(R_{2}-R\right) \sigma_{1} R_{1}+\left(R-R_{1}\right) \sigma_{2} R_{2}\right. \\
& +\frac{\sigma_{1} \sigma_{2}}{w}\left(R_{2}-R_{1}\right)\left(R_{1} R_{2}-w R\right) \\
& \left.+R_{1}\left(w+R_{2}\right)\left(R_{2}-R\right) \frac{\mathrm{d} \sigma_{1}}{\mathrm{~d} w}+R_{2}\left(w+R_{1}\right)\left(R-R_{1}\right) \frac{\mathrm{d} \sigma_{2}}{\mathrm{~d} w}\right] .
\end{aligned}
$$

Next, we can deduce that:

$$
\begin{aligned}
\frac{\mathrm{d} D}{\mathrm{~d} w}= & {\left[\left(\gamma w+R_{2}\right)\left(R_{2}-R\right) \sigma_{1} R_{1}+\left(\gamma w+R_{1}\right)\left(R-R_{1}\right) \sigma_{2} R_{2}\right] } \\
& \times \frac{f_{1}^{2}}{w}\left[\frac{f_{11}^{2} \sigma_{2} R_{2}}{f_{1}^{2}}+\sigma_{1}+\sigma_{2}\right]+f_{1}^{2}\left[\gamma\left\{\left(R_{2}-R\right) \sigma_{1} R_{1}+\left(R-R_{1}\right) \sigma_{2} R_{2}\right\}\right. \\
& +\frac{\sigma_{1} \sigma_{2}\left(R_{2}-R_{1}\right)}{w}\left(R_{1} R_{2}-w R\right)+R_{1}\left(\gamma w+R_{2}\right)\left(R_{2} \quad R\right) \frac{\mathrm{d} \sigma_{1}}{\mathrm{~d} w} \\
& \left.+R_{2}\left(\gamma w+R_{1}\right)\left(R-R_{1}\right) \frac{\mathrm{d} \sigma_{2}}{\mathrm{~d} w}\right] .
\end{aligned}
$$

Hence

$$
\begin{aligned}
D \frac{\mathrm{d} N}{\mathrm{~d} w}-N \frac{\mathrm{d} D}{\mathrm{~d} w}=\frac{D N}{w}\left[\frac{-w}{w+R_{1}}+\frac{\gamma w}{\gamma w+R_{2}}\right]+(\gamma-1) R_{1} R_{2} f_{1}^{1} f_{1}^{2} \\
\quad \times\left\{\sigma_{1}\left(R_{2}-R\right)+\sigma_{2}\left(R-R_{1}\right)\right\}\left\{\left(R_{2}-R\right) \sigma_{1} R_{1}+\left(R-R_{1}\right) \sigma_{2} R_{2}\right. \\
\left.\quad-R \sigma_{1} \sigma_{2}\left(R_{2}-R_{1}\right)\right\}-(\gamma-1) f_{1}^{1} f_{1}^{2}
\end{aligned}
$$




$$
\begin{aligned}
& \times\left\{\left(R_{2}-R\right) \sigma_{1} R_{1}+\left(R-R_{1}\right) \sigma_{2} R_{2}\right\} \sigma_{1} \sigma_{2} R_{1} R_{2}\left(R_{2}-R_{1}\right) \\
& -(\gamma-1)\left(R_{2}-R_{1}\right) w\left(R-R_{1}\right)\left(R_{2}-R\right) \\
& \times R_{1} R_{2} f_{1}^{2} f_{1}^{1}\left\{\sigma_{2} \frac{\mathrm{d} \sigma_{1}}{\mathrm{~d} w}-\sigma_{1} \frac{\mathrm{d} \sigma_{2}}{\mathrm{~d} w}\right\}
\end{aligned}
$$

Hence [ using $\frac{\mathrm{d} Q_{2}}{\mathrm{~d} w}=\frac{-D}{w\left(R_{2}-R_{1}\right)^{2}}$ ] we get :

$$
\begin{aligned}
\frac{\mathrm{d}^{2} Q_{1}}{\mathrm{~d} Q_{2}^{2}}= & \frac{-w\left(R_{2}-R_{1}\right)^{2}}{D^{2}}\left[\frac{N\left(\gamma R_{1}-R_{2}\right)}{\left(w+R_{1}\right)\left(\gamma w+R_{2}\right)}+\frac{(\gamma-1) R_{1} R_{2} f_{1}^{1} f_{1}^{2}}{D}\right. \\
& \times\left\{\left\{\left(R_{2}-R\right) \sigma_{1} R_{1}+\left(R-R_{1}\right) \sigma_{2} R_{2}-R \sigma_{1} \sigma_{2}\left(R_{2}-R_{1}\right)\right\}\right. \\
& \times\left\{\sigma_{1}\left(R_{2}-R\right)+\sigma_{2}\left(R-R_{1}\right)\right\} \\
& -\sigma_{1} \sigma_{2}\left(R_{2}-R_{1}\right)\left\{\left(R_{2}-R\right) \sigma_{1} R_{1}+\left(R-R_{1}\right) \sigma_{2} R_{2}\right\} \\
& \left.\left.-w\left(R_{2}-R_{1}\right)\left(R_{2}-R\right)\left(R-R_{1}\right)\left\{\sigma_{2} \frac{\mathrm{d} \sigma_{1}}{\mathrm{~d} w}-\sigma_{1} \frac{\mathrm{d} \sigma_{2}}{\mathrm{~d} w}\right\}\right\}\right]
\end{aligned}
$$

It should be obvious from (15) that it is difficult in general to determine the sign of $\mathrm{d}^{2} Q_{1} / \mathrm{d} Q_{2}^{2}$. One has therefore to consider special cases.

(1) If there is no differential (i.e. $\gamma=1$ ), then $\mathrm{d}^{2} Q_{1} / \mathrm{d} Q_{2}^{2}<0$ since the lengthy right-hand-side term in the bracket in (15) cancels out and $\left(R_{1}-R_{2}\right) N$ is always positive. Thus we get the standard result that the production possibility curve is concave to the origin.

(2) Where, however, $\gamma \neq 1$, we can show that the production possibility curve may have both convex and concave stretches. We can do this by evaluating $\mathrm{d}^{2} Q_{1} / \mathrm{d} Q_{2}^{2}$ at two extreme points: complete speciali- 
sation in $Q_{1}$ (so that $R_{1}=R$ ) and in $Q_{2}$ (so that $R_{2}=R$ ), and showing that the production possibility curve is concave at one end and convex at the other. Assuming that $\mathrm{d} \sigma_{i} / \mathrm{d} w$ is well-behaved for $i=1,2$, we can see from (15) that, quite generally:

$$
\begin{aligned}
& \frac{\mathrm{d}^{2} Q_{1}}{\mathrm{~d} Q_{2}^{2}}\left(\text { given } R_{1}=R\right)=\frac{-w\left(R_{2}-R\right)^{2}}{D^{2}} \\
& \left.\qquad \frac{N\left(\gamma R-R_{2}\right)}{(w+R)\left(\gamma w+R_{2}\right)}+\frac{(\gamma-1) R_{2} f_{1}^{1} f_{1}^{2}\left(R_{2}-R\right)^{2} \sigma_{1}^{2} R^{2}\left(1-2 \sigma_{2}\right)}{D}\right]
\end{aligned}
$$

$\frac{\mathrm{d}^{2} Q_{1}}{\mathrm{~d} Q_{2}^{2}}\left(\right.$ given $\left.R_{2}=R\right)=\frac{-w\left(R-R_{1}\right)^{2}}{D^{2}}$

$$
\left[\frac{N\left(\gamma R_{1}-R\right)}{\left(w+R_{1}\right)\left(\gamma w+R_{1}\right)}+\frac{(\gamma-1) R_{1} f_{1}^{1} f_{1}^{2}\left(R-R_{1}\right)^{2} \sigma_{2}^{2} R^{2}\left(1-2 \sigma_{1}\right)}{D}\right]
$$

Suppose now that $R_{1}(w)>R_{2}(w)$ for all $w$ in $[\underline{\mathbf{w}}, \bar{w}]$. Then $R_{1}(\underline{\mathbf{w}})=R=R_{2}(\bar{w})$ and $R_{1}(w)>R>R_{2}(w)$ for all $w$ in $(\underline{\mathbf{w}}, \bar{w})$. In this case, it follows that $N>0$ and $D<0$. Assume further the specific values: $\gamma>1$ and $\sigma_{1}(\bar{w}) \geq \frac{1}{2}$. Then clearly $\mathrm{d}^{2} Q_{1} / \mathrm{d} Q_{2}^{2}<0$ when $R_{2}=R$ (i.e. $w=\bar{w}$ ). If it so happens that when $R_{1}=R$ (i.e. $\left.w=\underline{\mathbf{w}}\right), \gamma R<R_{2}(\bar{w})$ and $\sigma_{2}(\underline{w}) \leq \frac{1}{2}$, then $\mathrm{d}^{2} Q_{1} / \mathrm{d} Q_{2}^{2}>0$. Thus the production possibility curve will be convex in the neighbourhood of one specialisation point and concave in the neighbourhood of the other.

(3) We may finally consider the case where $\sigma_{1}=\sigma_{2}$ equals a constant, showing that this can lead to a production possibility curve which is smoothly convex (to the origin) throughout. ${ }^{4}$ In this case of CES production functions, with identical elasticities for both industries, (15) reduces to:

$$
\begin{aligned}
\frac{\mathrm{d}^{2} Q_{1}}{\mathrm{~d} Q_{2}^{2}}= & \frac{-w\left(R_{2}-R_{1}\right)^{2}}{D^{2}} \\
& {\left[\frac{N\left(\gamma R_{1}-R_{2}\right)}{\left(w+R_{1}\right)\left(\gamma w+R_{2}\right)}+\frac{\sigma^{2}(\gamma-1) R_{1} R_{2} R\left(R_{2}-R_{1}\right)^{2} f_{1}^{1} f_{1}^{2}(1-2 \sigma)}{D}\right] }
\end{aligned}
$$

4 Kemp and Herberg have independently arrived at this conclusion for CES production functions. 
If we write $f^{i}=\left[\alpha_{i} R_{i}^{-\epsilon}+\left(1-\alpha_{i}\right)\right]^{-1 / \epsilon}$ (where $\sigma=1 /(\epsilon+1)$ ) then we get $R_{1}(w)=\left(\alpha_{1} /\left(1-\alpha_{1}\right)\right)^{\sigma} w^{\sigma}$ and $R_{2}(w)=\left(\gamma \alpha_{2} /\left(1-\alpha_{2}\right)\right)^{\sigma} w^{\sigma}$. This means that $R_{2}=\eta R_{1}$ where $\eta=(\beta \gamma)^{\sigma}$, and $\beta=\left(\alpha_{2} /\left(1-\alpha_{2}\right)\right) /\left(\alpha_{1} /\left(1-\alpha_{1}\right)\right)$. Since $\sigma>0, \eta \sum 1$ according as $\beta \gamma \gtreqless 1$. It can be shown that $N=-f_{1}^{1}(\eta-1) \sigma R_{1}\left(w R+\eta R_{1}^{2}\right)$ and $D=f_{1}^{2}(\eta-1) \sigma R_{1}\left(\gamma w R+\eta R_{1}^{2}\right)$. Substituting these into the expression for $\mathrm{d}^{2} Q_{1} / \mathrm{d} Q_{2}^{2}$ we get:

$$
\begin{aligned}
\frac{\mathrm{d}^{2} Q_{1}}{\mathrm{~d} Q_{2}^{2}}= & \frac{-w R_{1}^{4}(\eta-1)^{3} f_{1}^{1}}{D^{2}} \\
& \times\left[\frac{(\eta-\gamma)\left(w R+\eta R_{1}^{2}\right)}{\left(w+R_{1}\right)\left(\gamma w+\eta R_{1}\right)}+\frac{(\gamma-1)(1-2 \sigma) \eta R R_{1}}{\left(\gamma w R+\eta R_{1}^{2}\right)}\right] .
\end{aligned}
$$

It is clear from (16) that if $\alpha_{1}=\alpha_{2}$ and $\frac{1}{2} \leq \sigma \leq 1$ (i.e. when $\beta=1$ ), $\mathrm{d}^{2} Q_{1} / \mathrm{d} Q_{2}^{2}>0$ for all $\gamma \neq 1$. The reason is that, in this case, either $1>\eta>\gamma$ or $\gamma>\eta>1$. Thus the production possibility curve is convex throughout.

\section{APPENDIX: a numerical example}

The following numerical example demonstrates the possibilities of (a) multiple equilibria corresponding to a given commodity-price ratio,

(b) perverse comparative-static response to changes in this price ratio and (c) 'normal' response being associated with 'perverse' curvature of the production possibility curve.

Let $f^{1}=\left[{ }_{2}^{1} R_{1}^{-1}+\frac{1}{2}\right]^{-1}$ and $f^{2}=\left[\frac{1}{9} R_{2}^{\frac{1}{2}}+\frac{8}{9}\right]^{2}$. Let $\gamma=8$ and $R=4$. It is easy to deduce, using eqs. (6) and (7), that $R_{1}(w)=w^{\frac{1}{2}}$, $R_{2}(w)=w^{2}, \underline{\mathbf{w}}=2$ and $\bar{w}=16$. Hence $\left[\gamma R_{1}(w)-R_{2}(w)\right]=8 w^{\frac{1}{2}}-w^{2}$ and thus is positive in $\underline{\mathbf{w}}=2 \leq w<4$, zero when $w=4$ and negative in $4<w \leq 16=\bar{w}$. Of course $R_{2}(w)>R_{1}(w)$ for all $w$ in $(2,16)$. The resulting production possibility curve is presented in fig. 3 . This curve is convex throughout, as can be verified also by algebraic analysis.

In fig. 4, the function $p(w)$ is plotted. As was proved earlier (recall eq. (11)) and as is evident from the figure, $p(w)$ increases with $w$ when $\gamma R_{1}(w)>R_{2}(w)$, i.e. when $w$ is in the interval $(2,4)$ and decreases as $w$ increases when $\gamma R_{1}(w)<R_{2}(w)$, i.e. when $w$ is in the interval 


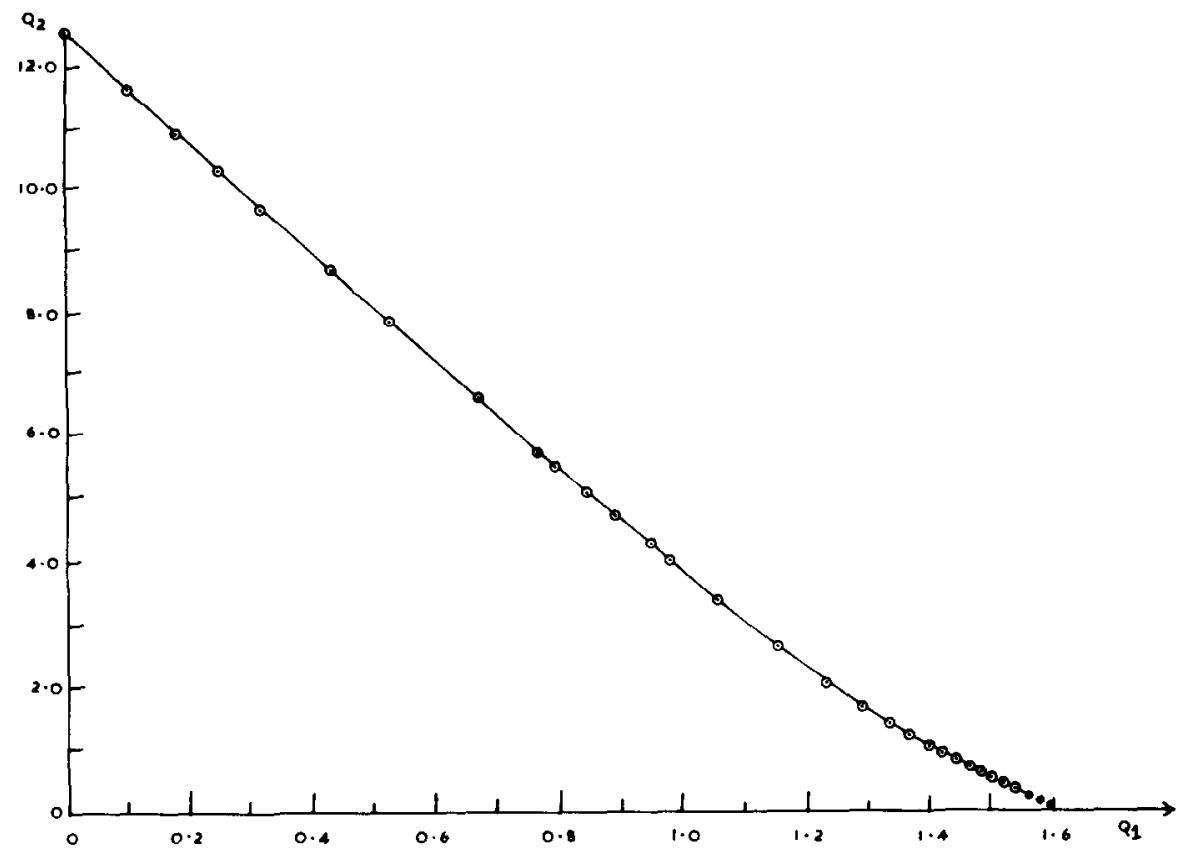

Fig. 3.

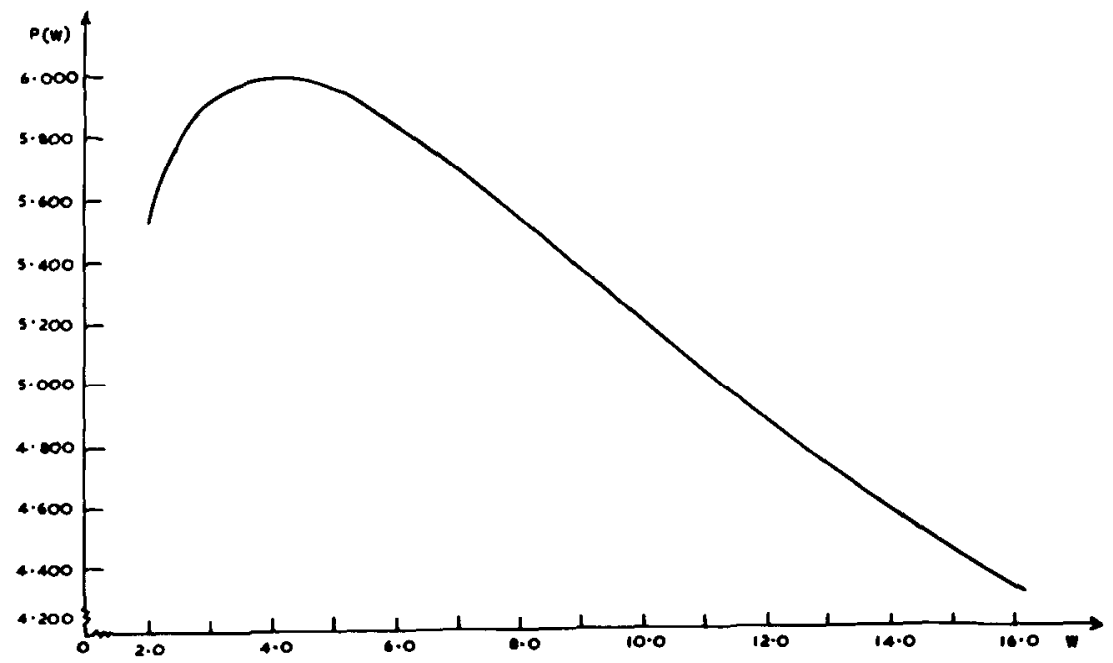

Fig. 4. 
$(4,16)$. Thus $p(w)$ starts from a value of about 5.56 when $w=2$, reaches a maximum of 6 when $w=4$, and declines steadily to a value of 4.32 when $w=16$. Thus it follows that if the commodity-price ratio happens to be anywhere in the range $5.56 \leq p<6$, there are two equilibrium values of $w$ corresponding to each such $p$, one in the interval $(2,4)$ and the other in the interval $(4,7.81)$. If either $4.32 \leq p<5.56$ or $p=6$, there is one and only one equilibrium value of $w$.

Next, note that with $R_{2}(w)>R_{1}(w)$ for all $w$ in $(2,16), \mathrm{d} Q_{2} / \mathrm{d} w<0$. Suppose we now increase $p$ from $p=4.32$ upto $p=5.56$. Then the equilibrium value of $w$ decreases steadily from $w=16$ to $w=7.81$ (approximately) and $Q_{2}$ increases steadily from $Q_{2}=0$ to $Q_{2}=0.66$ (approximately). Thus the response is 'normal', i.e. the equilibrium output of the second commodity is larger when its relative price is higher, even though the production possibility curve is convex to the origin.

\section{References}

Bhagwati, J. and V.K.Ramaswami, 1963, Domestic distortions, tariffs and the theory of optimum subsidy, J. Political Economy 71, 44-50.

Findlay, R. and H.Grubert, 1959, Factor intensities, technological progress and the terms of trade, Oxford Economic Papers 11, 111-121.

Fishlow, A. and P.David, 1961, Optimal resource allocation in an imperfect market setting, J. Political Economy 69, 529-546.

Hagen, E., 1958, An economic justification of protectionism, Quart. J. Economics 72, 496514.

Johnson, H., 1966, Factor market distortions and the shape of the transformation curve, Econometrica $34,686-708$. 